\title{
A Consideration of Effect of Sound Environments in Libraries
}

\author{
Hatsue WADA $^{\mathrm{a}^{*}}$, Riku Iida ${ }^{\mathrm{a}}$, Shuji Ogawa ${ }^{\mathrm{a}}$, Kazunori Miyamoto ${ }^{\mathrm{b}}$, Yukio MORI $^{\mathrm{a}}$ \\ ${ }^{a}$ Salesian Polytechnic, 4-6-8 Oyamagaoka, Machida-City, Tokyo, 194-0215,Japan \\ ${ }^{\mathrm{b}}$ Kyushu Women's University, 1-1, Jiyugaoka, Yahatanishi-ku, Kitakyushu-shi, Fukuoka, 807-8586 Japan \\ *Corresponding Author: wada@ salesio-sp.ac.jp
}

\begin{abstract}
In previous studies, the genuinely silent sound environment was created using sound masking ${ }^{(1)}$ at a Polytechnic College library for commons. In this research, one user commented: "I can concentrate more on the music environment than in the completely silent environment." From that comment, we suggest that the possibilities are as follows. First, control of the sound environment changes a library into a "place" to realize a variety of utilizations. Next, sound environment that is "comfortable" for users improves their concentration and eventually promotes educational and research effects in library utilization. Therefore, we investigate recognition of the sound environment of libraries ${ }^{(2)}$. The results, confirm the following trends regarding recognition of Background Music (BGM) in college libraries.

- BGM has the effect of enhancing concentration and relaxation for personal learning and reading.

- Music that is focused on melody and harmony is preferable as BGM, whereas music focused on vocals is dispreferred.
\end{abstract}

At the same time, the correlation with taste in music and other individual characteristics is unclear. Therefore, we conducted listening experiments of two kinds of tasks (reading and calculating) in libraries in order to objectively verify these correlations.

From these results, we confirmed the following trends.

- It was easier to do tasks, regardless of which task they were doing, when the BGM was played.

- In the reading task, even though the experiment subjects felt it was easier to perform the task, the task was not accelerated.
- In the calculating task, listening to BGM made the subjects feel the task was easier and the work was accelerated.

In this paper, we describe the results of listening experiments and consideration of a creating method for better sound environments in libraries.

Keywords: .Library, Sound Environments

\section{Introduction}

A quiet sound environment has long been considered desirable in libraries. However, in recent years, the purpose of libraries established along with other facilities for local communities has diversified. Therefore, it is no longer correct to say that a quiet environment is essential. To resolve the above problem, noise measures, such as the introduction of sound masking, have been adopted for the sound environments of libraries. The possibility of providing sound environments to achieve library environments has also been suggested, where, in addition to noise countermeasures, people can relax ${ }^{(1)}$. A questionnaire survey, conducted in relation to sound environments in libraries, has confirmed that people feel it is better to have a sound environment while reading or doing homework. ${ }^{2)}$ In particular, users were conscious of how such an environment relaxes them or improves their ability to concentrate while reading or during personal study. However, the correlation of such consciousness with the actual amount of work done is not verified and requires objective confirmation. Therefore, this paper reports on listening experiments conducted in libraries with the help of two kinds of tasks, in order to objectively verify these correlations. 


\section{Listening Experiment}

In this section, we show the methodology of the listening experiment.

(a) Purpose

As a result of the preliminary investigation, BGM was found to have a "relaxation" effect and an ability to "promote concentration" during tasks performed in libraries such as reading and studying. As such, this study clarifies the following items with the aim of developing better sound environments.

- The relationship between the influence of the sound environment on consciousness and the amount of work in two tasks in libraries.

\section{(b) Participants}

The experiment subjects consisted of 13 students (all male) of a Polytechnic College; ten 5th grade students and two 4th grade students and one first grade student of advanced course were asked to participate in the study in advance. (c) Experiment period

October 2, 2017 Reading Task

October 9, 2017 Calculating Task

\section{(d) Experiment method}

We set up speakers around the reading space in the library and played environmental BGM music. We created a sound environment in which the volume of the music was at a slightly higher level than the background noise of the library. We show the layout of the library in Fig. 1.

Then, three kinds of BGM - Classical, Jazz, and Healing - were played during the experiments. As a control, a fourth case, 'no BGM', was set. Table 1 shows the three sound sources used for the experiments. The piece called of big wave in the Japan Sea, which is Healing music, includes $1 / f$ fluctuation. Musha clarified that $1 / f$ fluctuation bring to relaxing, and therefore it was set as a sound source in anticipation of this effect ${ }^{(3)}$.

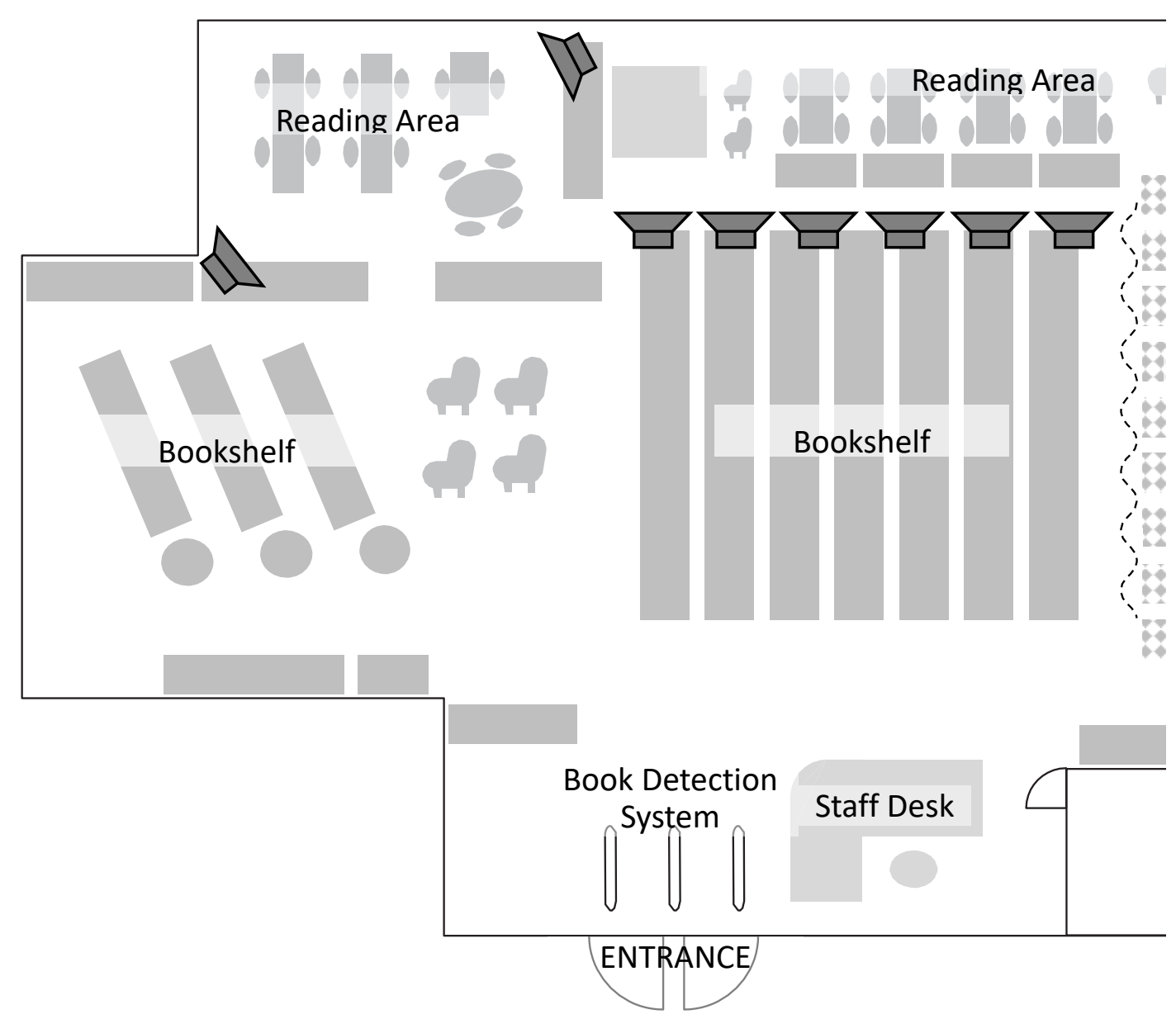

Fig. 1 The layout of the library. 
Table 1. Sound sources used for experiments.

\begin{tabular}{|c|c|c|}
\hline Genre & Artist & Title \\
\hline Classical & J.S.Bach & $\begin{array}{c}\text { Air on the G String } \\
\text { from Suites for orchestra No.3 }\end{array}$ \\
\hline Jazz & Miles Davis & Violets For Your Furs \\
\hline $\begin{array}{l}\text { Healing } \\
\text { music }\end{array}$ & $\begin{array}{l}\text { Supervised by } \\
\text { M.Nakagawa }\end{array}$ & Sound of big wave in the Japan Sea \\
\hline
\end{tabular}

The two tasks performed for the experiments were reading and calculating. The reading task phase was planned by first preparing paperbacks and novels from the library's collection and selecting those according to the tastes of the experiment subjects. The test subjects recorded the number of pages they read every three minutes to obtain the amount of work done. The calculating task was carried out using a Kraepelin test, which had the test subjects add adjacent rows of numbers, arranged horizontally. Similar to the reading task, the experiment subjects indicated the total amount of work completed every three minutes.

The experiment sequence is shown in Fig. 2 and 3. The letters on the figure signify four conditions:

$$
\begin{aligned}
& \text { - A: no BGM (control) } \\
& \text { - B: Classical } \\
& \text { - C: Jazz } \\
& \text { - D: Healing }
\end{aligned}
$$

The inverted triangles in the figure record during which BGM - the type played in the first half or the type played in the last half - it was easier for each experiment subject to perform his task. The BGM was played for the experiment subjects in a way that it would not be the same during the reading task and the calculating task. Each was played for three minutes to permit a paired comparison method of all four conditions.

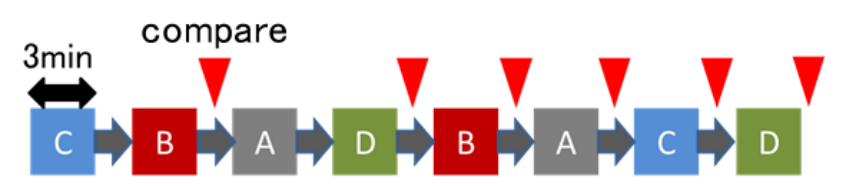

Fig. 2. The Sequence of reading task.

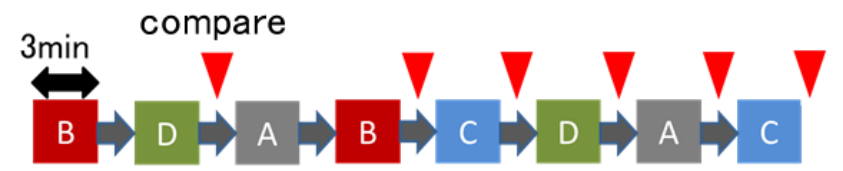

Fig. 3. The Sequence of calculating task.

\section{Listening experiment results}

\subsection{The influence of the sound environment on consciousness}

Fig. 4 and 5 show the scale value of each BGM ranked by Thurstone method. The higher scale value, the easier it was for the subjects to perform their object tasks (reading and calculating). The figures confirmed that the subjects felt it was easier to do tasks, regardless of which task they were doing, when the BGM was played. This supports the results of past research.

\subsection{The correlation of the influence of the sound environment on consciousness and amount of work}

Fig. 6 and 7 show the correlation between the amount of work and scale values. Fig. 5 shows that in the reading task case, even though the experiment subjects felt it was easier to perform the task, the task was not accelerated. On the other hand, Fig. 6 shows that during the calculating task, listening to BGM made the subjects feel the task was easier and the work was accelerated.

From above, these are actually very interesting results and are evaluated as follows: Among the sound sources used for the experiment, Classical and Healing music had relatively simple melodies, while Jazz had a relatively complex melody. This means that the simple melodies accelerated the calculating task, and the complex melody accelerated the reading task. As for the qualities of the tasks, it is said that reading is a task that expands a person's imagination, whereas calculating is a task that involves the free use of mathematical logical thinking. In addition, reading is a right-brain task and calculating is a left-brain task. Therefore, one can presume that there are some correlations between the quality of a task and the type of BGM. However, it is too early to reach this conclusion

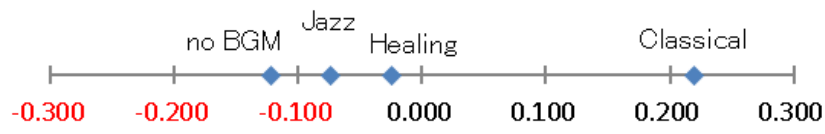

Fig. 4. Scale values in reading task.

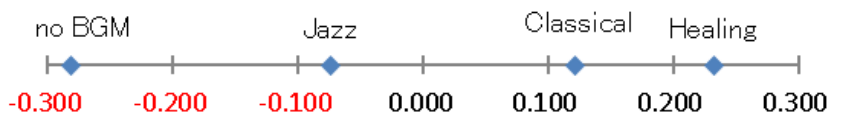

Fig. 5. Scale values in calculating task. 


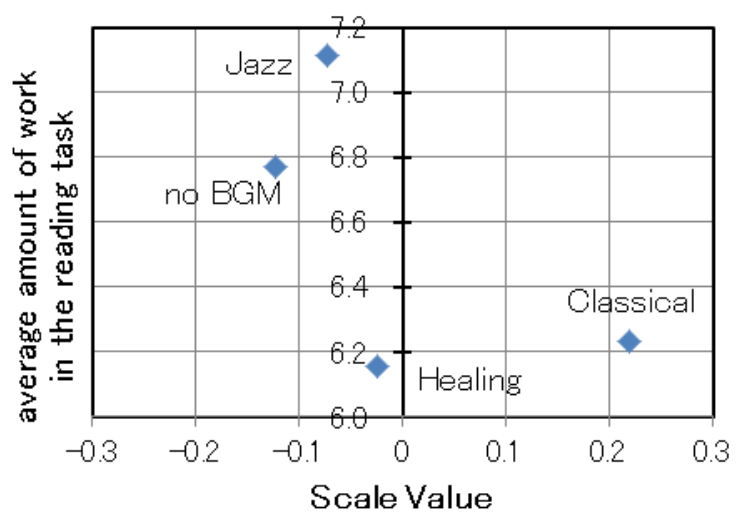

Fig. 6. Average amount of work in the reading task.

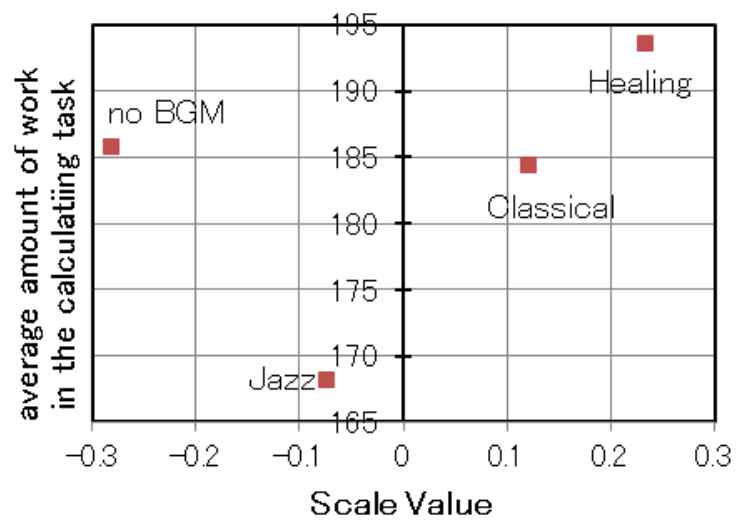

Fig. 7. Average amount of work in the calculating task.

based on this experiment only, and therefore further verification is needed.

\section{Conclusion}

In this paper, we described the results of the listening experiment which conducted to enhance the educational effectiveness of libraries by verifying the correlation between the acceleration of the task performance and the sound environment in libraries.

From these results, it has been clarified that there is a correlation of some kind between the quality of a task and the type of BGM played as the sound environment.

Further studies, we will conduct to concretely describe these correlations. At the same time, because these experiments were conducted with experiment subjects of limited ages and sex, it will be necessary to also verify in the future whether or not age or sex affects the results.

\section{References}

(1) Hatsue Wada : "Trial of learning commons which supports active learning in creative education, https://kaken.nii.ac.jp/ja/grant/KAKENHI-PROJECT-1 $\underline{6 \mathrm{H} 00087 /}$ as of June 10, 2018 (in Japanese)

(2) Hatsue WADA, Yukio MORI : "Study of sound environments to effectively facilitate user's learning and research in college libraries", Proc. of ICISIP 2017, Hawaii, USA, pp.345-348, 2017

(3) Toshimitsu Musha: "The world of fluctuation (yuragi no sekai)", Kodansha, Tokyo, Japan, 1980 (in Japanese) 